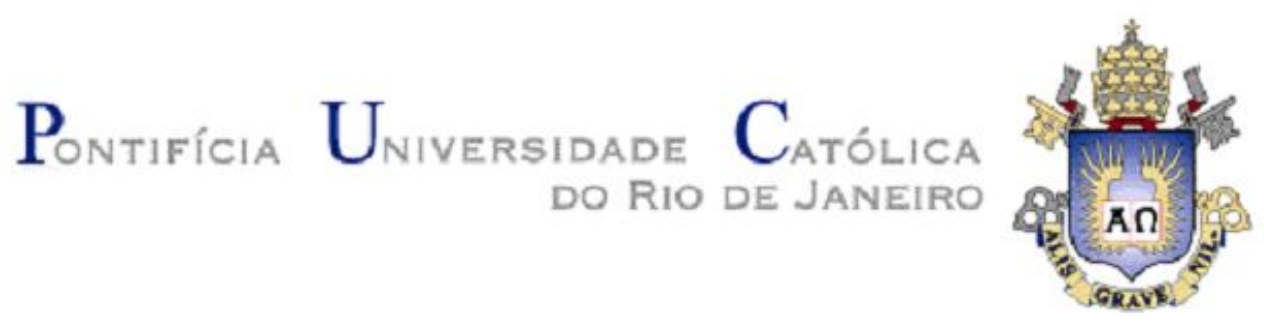

Eider Fernando Alves dos Santos

Desenvolvimento de método para determinação multielementar em biodiesel por ICP-MS

\begin{abstract}
Dissertação de Mestrado
Dissertação apresentada como requisito parcial para obtenção do título de Mestre pelo Programa de Pós-Graduação em Química do Departamento de Química da PUC-Rio.
\end{abstract}

Orientadores: Profa. Tatiana Dillenburg Saint'Pierre Co-Orientadora: Profa. Roseli Martins de Souza 


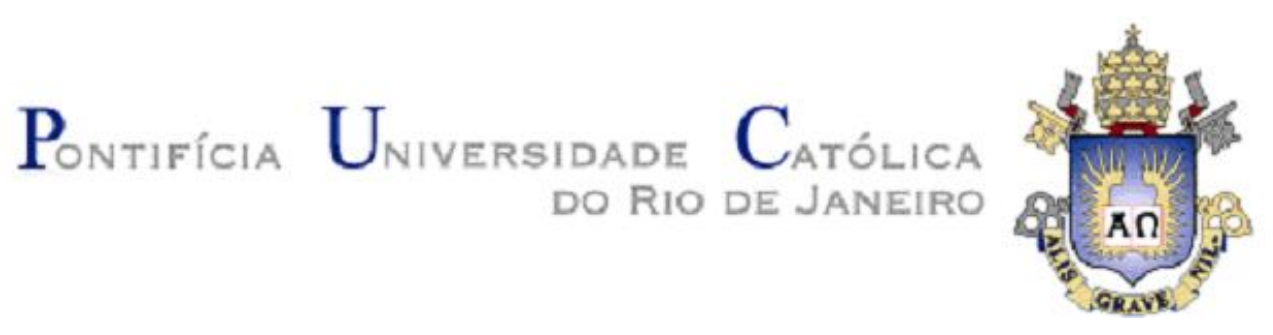

Eider Fernando Alves dos Santos

\title{
Desenvolvimento de método para determinação multielementar em biodiesel por ICP-MS
}

\begin{abstract}
Dissertação apresentada como requisito parcial para obtenção do título de Mestre pelo Programa de Pós-Graduação em Química do Departamento de Química da PUC-Rio. Aprovada pela Comissão Examinadora abaixo assinada.
\end{abstract}

\author{
Profa. Tatiana Dillenburg Saint'Pierre \\ Orientador \\ Departamento de Química - PUC-Rio \\ Profa. Roseli Martins de Souza \\ Co-orientadora \\ Departamento de Química - PUC-Rio
}

Profa. Adriana Gioda

Departamento de Química - PUC-Rio

Prof. Fabio Grandis Lepri

UFF

Prof. José Eugenio Leal

Coordenador(a) Setorial do Centro Técnico Científico - PUC-Rio

Rio de Janeiro, 27 de abril de 2011 
Todos os direitos reservados. É proibida a reprodução total ou parcial do trabalho sem autorização da universidade, do autor e do orientador.

Eider Fernando Alves dos Santos

Graduou-se em Licenciatura em Química com atribuições tecnológicas pela UFRJ em 2003. Pós-graduado em Ciências Ambientais pelo Instituto de Biologia da UFRJ e com 15 anos de experiência em diferentes segmentos da indústria química.

Ficha Catalográfica

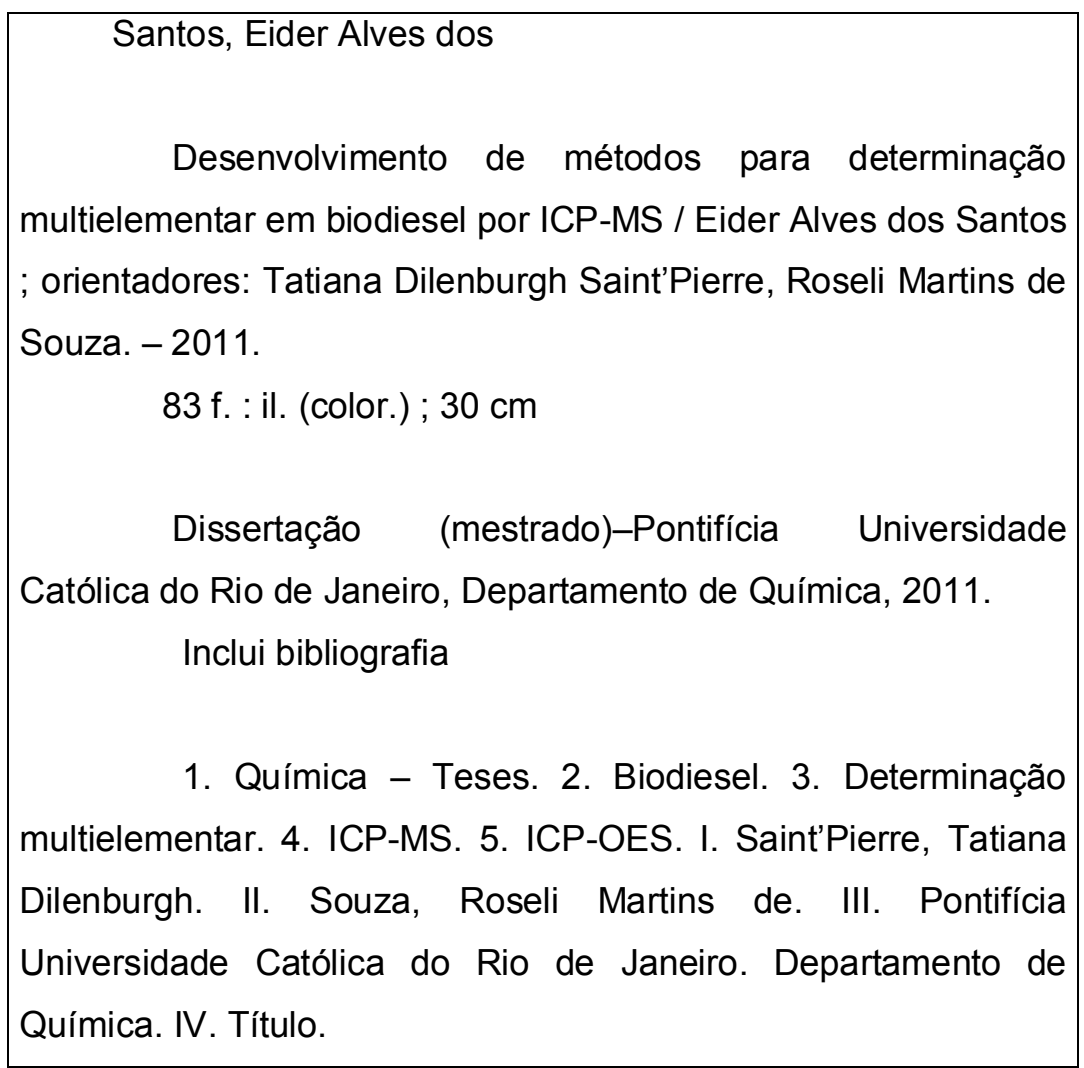

CDD: 540 
A DEUS, por tudo de maravilhoso que me proporciona. 


\section{Agradecimentos}

Às minhas orientadoras, Profa. Tatiana Dillenburg Saint'Pierre e Profa. Roseli Martins de Souza, pela orientação, estímulo e amizade durante a realização deste trabalho.

À minha esposa, pelo suporte e compreensão constantes.

Aos meus pais, José e Gricelha, pela educação e suporte.

Aos técnicos André Vechi, Maurício Dupim e Álvaro Pereira, pelo suporte, paciência e auxílio competente e dedicado que sempre dispensaram a mim.

A todos os meus amigos da PUC-Rio, mas especialmente ao Jefferson Rodrigues de Souza por todo auxílio.

À PUC-Rio, pela disponibilidade de vagas para alunos bolsistas, aos professores do Departamento de Química da PUC-Rio, pela contribuição na minha formação acadêmica e à banca examinadora, pela participação e contribuição para esse trabalho.

À Petrobrás, pelo suporte financeiro dado sob a forma de equipamentos e amostras. 


\section{Resumo}

Santos, Eider Fernando Alves; Saint'Pierre, Tatiana Dillenburb. Desenvolvimento de método para determinação multielementar em biodiesel por ICP-MS. Rio de Janeiro, 2011. 83p. Dissertação de Mestrado Departamento de Química, Pontifícia Universidade Católica do Rio de Janeiro.

Os elementos de maior interesse no biodiesel são os resíduos de catálise do processo produtivo ( $\mathrm{Na}$ e K), os delimitados por legislação ambiental vigente (S, por exemplo) e os elementos venenosos aos catalisadores automotivos ( $\mathrm{Pb}, \mathrm{V}, \mathrm{P}$ e $\mathrm{Hg}$ ); porém, pesquisas orientadas no sentido de produzir metodologias analíticas para a realização de determinações em biodiesel vêm buscando um mapeamento elementar mais completo do combustível. Técnicas como ICP OES, ICP-MS com célula de reação/colisão, FAAS, GFAAS têm sido usualmente empregadas em determinações diretas ou não, mas ainda existem combinações de preparo de amostra e técnica analítica a ser pesquisadas. O presente trabalho teve como objetivo a avaliação de um sistema de decomposição ácida de amostra de biodiesel, visando à decomposição ácida da maior quantidade possível de matéria orgânica presente na matriz e a consequente determinação em ICP-MS. O processo de decomposição ácida, avaliado pela concentração de carbono residual, determinado em ICP OES, não requer grandes quantidades nem de energia térmica e nem do agente oxidante e ainda permite, após processo de redução da matriz nítrica remanescente, uma determinação direta em ICP-MS utilizando padrões aquosos na confecção das curvas e sem a necessidade de célula de colisão/reação. Foram determinados $\mathrm{Ag}, \mathrm{Ba}, \mathrm{Cu}$, $\mathrm{Mn}, \mathrm{Mo}, \mathrm{Ni}$, Ti e $\mathrm{Zn}$ em amostras de biodiesel de 4 matérias-primas diferentes e em duas amostras certificadas de biodiesel (NIST) e, tanto os resultados de recuperação, que variaram entre 90 e $110 \%$, quanto o comparativo realizado com as metodologias por ICP OES, validaram os resultados.

\section{Palavras-chave}

Biodiesel; determinação multielementar; ICP-MS; ICP OES 


\section{Abstract}

Santos, Eider Fernando Alves; Saint'Pierre, Tatiana Dillenburg (Advisor). Development of a method for multielemental determination in biodiesel by ICP-MS. Rio de Janeiro, 2011. 83p. MSc. Dissertation - Departamento de Química, Pontifícia Universidade Católica do Rio de Janeiro.

Biodiesel appeared in this scenario as an alternative short-term, compatible with the current technologies and without the necessity of major modifications. The elements of greatest interest are the residue of catalyst of the production process $(\mathrm{Na}, \mathrm{K})$, those limited by environmental regulations $(\mathrm{S})$ and those that act like poisons for the catalyst system of vehicles $(\mathrm{Pb}, \mathrm{V}, \mathrm{P}$ and $\mathrm{Hg}$ ), but researches aimed to produce analytical methodologies for determinations in Biodiesel, have sought to achieve a multielement mapping of the fuel (Woods ET AL, 2007). Techniques like ICP OES, ICP-MS with reaction cell, FAAS, GFAAS have been usually used in direct or indirect determinations, but still there are combinations of sample preparation and analytical techniques to be studied. This present work sought to evaluate an analyzing system in ICP-MS after a digestion procedure looking for the minimal quantity of organic matter at the final sample. The preliminary results showed that the digestion process, evaluated by the concentration of residual carbon determined by ICP OES, it can be fast and does not require large amounts of energy and oxidizing agent. And still allow, after reducing the nitric matrix, the use of aqueous standards at the calibration curve. It was monitored $\mathrm{Ag}, \mathrm{Ba}, \mathrm{Cu}, \mathrm{Mn}, \mathrm{Mo}, \mathrm{Ni}, \mathrm{Ti}$ and $\mathrm{Zn}$ in 4 different sources of raw material for biodiesel, and the method was validated by the analysis of two NIST standards by the comparison with ICP OES results and by the recover results ranged between 90 and $110 \%$.

\section{Keywords}

Biodiesel; multielemental determination; ICP-MS; ICP OES. 


\section{Sumário}

1. Introdução 14

1.1. Biodiesel 14

1.2. Determinação de metais em biodiesel 19

1.2.1. Espectrometria atômica 21

1.2.1.1. Espectrometria de absorção atômica (AAS) 24

1.2.1.2. Espectrometria de emissão óptica com plasma indutivamente acoplado (ICP OES) 26

1.2.1.3. Espectrometria de massas com plasma indutivamente acoplado (ICP-MS) 34

1.3. Análise de amostras orgânicas por técnicas com ICP 42

2. Objetivos $\quad 47$

$\begin{array}{ll}\text { 2.1. Objetivos específicos } & 47\end{array}$

3. Materiais e Métodos $\quad 48$

3.1. Reagentes, soluções e amostras 48

3.2. Equipamentos 49

3.2.1. ICP OES 49

3.2.2. ICP-MS DRC II

3.2.3. Forno de micro-ondas 52

3.3. Metodologias 53

3.3.1. ICP-MS: Decomposição ácida das amostras em forno de micro-ondas 53

3.3.2. Medida das amostras de biodiesel por ICP-MS 54

3.3.3. Medida das amostras de biodiesel por ICP-OES 55

3.3.3.1. ICP OES: Diluição em etanol

3.3.3.2. ICP OES: Diluição em QAV 58

3.3.3.3. ICP OES: Diluição em xileno $\quad 59$

3.3.4. Parâmetros de mérito 60

3.3.5. Limite de detecção (LD) 60

3.3.6. Sensibilidade 61

3.3.7. Linearidade - coeficiente de correção $\left(R^{2}\right) \quad 61$ 
3.3.8. Robustez

61

3.3.9. Intervalo de confiança (IC)

4. Resultados e Discussão

63

4.1. ICP-MS: Decomposição ácida das amostras de biodiesel 63

4.1.1. Otimização do programa do forno de micro-ondas 63

4.2. Otimização dos parâmetros instrumentais do ICP-MS 68

4.3. Parâmetros de mérito obtidos com o método proposto, por ICP-MS 69

4.4. Parâmetros de mérito obtidos com os métodos por ICP OES 71

4.5. Análise das amostras de biodiesel por ICP OES e ICP-MS 73

4.6. Validação

$\begin{array}{ll}\text { 5. Conclusões } & 79\end{array}$

$\begin{array}{lr}\text { 6. Referências Bibliográficas } & 80\end{array}$ 


\section{Lista de figuras}

Figura 1 Efeito estufa (www.cidadeverde.com acessado em fevereiro 2011) 15

Figura 2 Reação geral de transesterificação (Química Nova, 30 (5): 2007) 17

Figura 3 Principais fontes de oleaginosas para fabricação de biodiesel no

Brasil 18

$\begin{array}{ll}\text { Figura } 4 \text { Evolução da produção de biodiesel no Brasil } & 18\end{array}$

Figura 5 Diagrama de Jablonsky de níveis de energia 23

Figura 6 Nebulizadores concêntrico de fluxo paralelo e de fluxo cruzado $\quad 27$

Figura 7 Desintegração do filme líquido ocorrida na ponta dos nebulizadores 28

Figura 9 Gráfico comparativo de primeiros potenciais de ionização 30

Figura 10 Processos sofridos pela amostra dentro do plasma 31

Figura 11 Interferência espectral em ICP OES do Cu sobre a linha do P 33

Figura 12 Interface do ICP-MS indicando os cones de amostragem e skimmer 36

Figura 13 Expansão gasosa sofrida pelo feixe após passagem pelo $\begin{array}{ll}\text { cone de amostragem } & 37\end{array}$

Figura 14 Esquematização do funcionamento do quadrupolo 38

Figura 15 Representação gráfica das trajetórias estáveis no quadrupolo 39

Figura 16 ICP OES - Labspectro PUC-Rio $\quad 50$

Figura 17 DRC-ICP-MS - Labspectro / PUC-Rio 51

Figura 18 Forno de micro-ondas Speedwave Four - Labspectro / PUC-Rio 52 


\section{Lista de tabelas}

Tabela 1 Especificação requerida do biodiesel segundo a ANP

Tabela 2 Exemplos de interferentes poliatômicos e por óxidos

que ocorrem comumente em ICP-MS

Tabela 3 Interferentes derivados da presença de carbono no plasma em ICP-MS

Tabela 4 Programa desenvolvido para a decomposição ácida das a mostras de biodiesel em micro-ondas.

Tabela 5 Condições experimentais para determinação de metais em biodiesel via ICP-MS

Tabela 6 Configurações do ICP OES para análise de biodiesel após diluição em solvente orgânico.

Tabela 7 Linhas espectrais utilizadas nas determinações realizadas em biodiesel após diluição em etanol, QAV e xileno.

Tabela 8- Condições operacionais empregadas na análise de biodiesel por ICP OES no Laboratório de análise espectrométricas da PUC-Rio, seguindo a NBR 15553.

Tabela 9 Passos da decomposição ácida de amostras de óleo vegetal sugeridos pelo fabricante do equipamento de micro-ondas.

Tabela 10 Variáveis utilizadas no planejamento estatístico fatorial para determinação do método de decomposição ácida das amostras de biodiesel. 65 Tabela 11 Experimentos de decomposição ácida de biodiesel em micro-ondas e suas respectivas respostas

Tabela 12 Condições otimizadas da etapa de decomposição ácida do programa do forno de micro-ondas para amostras de biodiesel e resultados dos parâmetros de avaliação.

Tabela 13 Parâmetros de mérito obtidos aplicando a metodologia em ICP-MS

Tabela 14 Parâmetros de mérito das metodologias de análise de amostras de biodiesel diluídas em diferentes solventes, por ICP OES. 
Tabela 16 Concentrações informados dos elementos traço presentes nos MRCs fornecidos pelo NIST.

Tabela 17 Resultados da leitura dos MRCs de biodiesel em ICP OES, após diluição direta em etanol. 


\section{Lista de Abreviaturas}

AAS - Espectrometria de absorção atômica

ABNT - Associação brasileira de normas técnicas

ANP - Agência Nacional do Petróleo, Biocombustíveis e derivados

ASTM - American Society for Testing and Materials

CNPE - Conselho nacional de política energética

CS AAS - Espectrometria de absorção atômica com fonte contínua

GF AAS - Espectrometria de absorção atômica com forno de grafite

ICP-MS - Espectrometria de massa com plasma indutivamente acoplado

ICP OES - Espectrometria de emissão óptica com plasma indutivamente acoplado

INMETRO - Instituto nacional de metrologia, normalização e qualidade industrial

LD - Limite de Detecção

MRC - Material de referência certificado

NBR - Denominação de norma técnica brasileira da ABNT

NIST - National institute of standards and technology 